\title{
1 Economic Burden of Hypoglycaemia among Type II Diabetes Mellitus Patients in
}

\section{Malaysia}

3 Syed Mohamed Aljunid ${ }^{1,2}$, Yin New Aung ${ }^{1}$, Aniza Ismail ${ }^{1}$, Siti Athirah Zafirah Abdul Rashid ${ }^{1}$, Amrizal M

$4 \quad$ Nur $^{1}$, Julius Cheah ${ }^{3,4}$ and Priya Matzen ${ }^{3,5}$

$5{ }^{1}$ International Centre for Casemix and Clinical Coding, Faculty of Medicine, UniversitiKebangsaan

6 Malaysia

$7 \quad{ }^{2}$ Department of Health Policy and Management, Faculty of Public Health, Kuwait University

$8{ }^{3}$ Market Access and Public Affairs, Novo Nordisk Pharma (Malaysia) SdnBhd, Kuala Lumpur, Malaysia

$9 \quad{ }^{4}$ Global Public Health, Jeffrey Cheah School of Medicine and Health Sciences, Monash University

10 Malaysia, Malaysia

$11{ }^{5}$ Human Development and Health, Faculty of Medicine, University of Southampton, UK

12

13 Corresponding Syed Mohamed Aljunid

14 E-mail: saljunid@gmail.com 
Abstract

\section{Objective}

21 The main aim of this study is to identify the direct cost and economic burden of hypoglycaemia among

22 patients with Type II diabetes mellitus in Malaysia.

\section{Methods}

24 The incurred cost for hypoglycaemia among patients admitted to University Kebangsaan Malaysia Medical

25 Centre (UKMMC) was explored from a cross sectional study. 20-79 year patients discharged between Jan

262010 to Sept 2015 and having an ICD-10 code of hypoglycaemia as a primary diagnosis in the casemix

27 database were included in the study. Costing analysis from the perspective of health providers was

28 completed using step-down approach. Data related to hospital cost were collected using hospital-costing

29 template, based on three levels of cost centres. The costing data from UKMMC was used to estimate the

30 national burden of hypoglycaemia among type II diabetics for the whole country.

\section{$31 \quad$ Results}

32 Of 244 diabetes patients admitted primarily for hypoglycaemia to UKMMC, 52\% were female and $88 \%$

33 were over 50 years old. The cost increased with severity. Managing a hypoglycaemic case requires 5 days

34 (median) of inpatient stay with a range of 2-26 days; and costs RM 9,083 (USD 2,323). 30\% of the cost

35 came from the ward services cost (final cost centre), 16\% of the cost came from ICU services and 15\%

36 from pharmacy services(secondarylevel cost centres). Based on the prevalence of hypoglycaemia-related

37 admissions of $3.2 \%$, the total cost of care for hypoglycaemia among adult diabetes in Malaysia is estimated

38 to be RM 117.4 (USD 30.0) million, which is translated as $0.5 \%$ of Ministry of Health budget.

\section{Conclusion}

40 Hypoglycaemia imposes substantial economic impact even without the direct and indirect cost incurred by

41 patients and other cost of complications. Proper diabetic care and health education is needed in diabetic

42 management to reduce episodes of hypoglycaemia. 


\section{Introduction}

48 Hypoglycaemia is a not an uncommon presentation at accident and emergency (A\& E) department. An

49 estimate of $2-4 \%$ of diabetes deaths is due to hypoglycaemia [1]. Hypoglycaemic episode is inevitable in

$5090 \%$ of diabetics with insulin therapy[2], and those with certain antihyperglycaemic medicine are also at

51 risk. Elderly and those with comorbid conditions are more likely to get severe hypoglycaemia. [3] The

52 incidence of hypoglycaemia varies with studies. On average, type 1 diabetics had two symptomatic

53 hypoglycaemia episodes per week [4]and one severe hypoglycaemia episode per year[5]; with a lesser

54 occurrence among type II diabetes patients.[2] However, type II diabetes with progressive insulin

55 deficiency, long duration of diabetes, and tight glycaemic control also have similar risk of hypoglycaemia

56 as type 1 diabetes.[5]

58 Eleven percent of type 2 diabetic patients with different anti-diabetic regimens suffered one or more

59 hypoglycaemic episodes per year. Miller et al reported that $24.5 \%$ of patients reported at least one

60 hypoglycaemic episode in 3 months period [6,7]. Mild hypoglycaemic attacks occurs frequently, as high as

61 1-2 episodes per week[8] and severe hypoglycaemic attacks occurs less frequently with an incidence rate of

621 to 2.7 episodes by per patient per year $[8,9]$.

$64 \mathrm{DM}$ is one of the commonest chronic non-communicable diseases globally. Its incidence and prevalence

65 are escalating, and Asia-Pacific region is at the forefront of the current epidemic. As of 2015, it is estimated

66 that $8.8 \%$ of adult population in Southeast Asia region has diabetes [10]. Prevalence of DM among

67 Malaysian over 20-79 years is estimated to be 17.5\%[11], ranking third in Asia-Pacific region. [12, 13] It

68 was estimated that in 2030, Malaysia would have a total number of 2.48 million diabetics compared to 0.94

69 million in 2000 that can be translated as $164 \%$ increase [14].

71 The chronic nature of diabetes and its devastating complications make it a very costly disease. To prevent

72 the risk of acute and chronic complication, diabetic patients required continuous medical care [2, 15].

73 Hypoglycaemia is unpredictable and undesirable side effects among diabetic patients. Frequent and 
74 potentially fatal complication may occur among hypoglycaemic patients with Type 1 or Type 2 diabetes treated with insulin, and in patients with Type 2 diabetes treated with certain oral anti-diabetic medicine.

77 Hypoglycaemic attacks require defence mechanism against falling serum glucose, and frequent attack result

78 in increasing cycles of recurrent hypoglycaemia [13]. Fear of hypoglycaemia is not uncommon in patients

79 with diabetes. Experiences of severe hypoglycaemic episodes increase the fear for future hypoglycaemic

80 event. Approximately $40 \%$ of patients admitted that fear of hypoglycaemia caused them to maintain their

81 blood glucose levels at higher than recommended values [14]. This questions compliance of anti-diabetic

82 medicine with possible complication due to uncontrolled diabetes and increased health care cost.

83 Regardless of bearing a significant burden by hypoglycaemia, the cost of hypoglycaemia in Malaysia is yet

84 known. This study identifies the direct cost and economic burden of hypoglycaemia among patients with

85 type II diabetes mellitus on insulin in Malaysia.

\section{$87 \quad$ Methods}

89 A cross sectional study was conducted to identify the incurred cost due to hypoglycaemia among patients

90 admitted to Universiti Kebangsaan Malaysia Medical Centre (UKMMC). Using the electronic medical

91 database kept at UKMMC, patients discharged from January 2010 to September 2015 were classified into

92 diagnosis related groups (DRG) with MY-DRG® grouping software. 20-79 year old patients having

93 hypoglycaemia as a primary reason for admission in the casemix database is identified using ICD 10 codes.

94 The ICD 10 codes associated with hypoglycaemia: E16.0 (Drug Induced Hypoglycaemia without coma,

95 E16.1 (Other Hypoglycaemia) and E16.2 (Hypoglycaemia Unspecified) were included in the study.

96 Costing analysis was carried out using step-down approach. Financial staffs in the hospital were given a

97 costing template to retrieve financial data from the hospital records. The templates classifies the

98 departments into three levels of cost centres: overhead cost centres (e.g.; administration, utilities,

99 maintenance etc.), intermediate cost centres (e.g.; pharmacy, radiology etc.) and final cost centres (all

100 wards and all clinics). Information on financial expenditures and output for each cost centre was recorded.

101 The information recoded includes the total expenditure, total number discharges, inpatient days, number of 
102 patient visits for outpatient clinics and floor space. All the information of the activities reflecting the

103 workload such as number of discharges, inpatient days, floor space and number of outpatient visits were

104 gathered for appropriate allocation.

106 Both capital cost (building, equipment and furniture cost) and recurrent cost (staff salary and other

107 recurrent cost) were combined in estimating the cost for each cost centre. The final allocated costs for each

108 inpatient cost centreswere then divided by the total units of inpatient days to obtain the cost of providing

109 services on a per-patient per-day of stay basis, which is referred as unit cost. The unit cost is finally

110 multiplied with the individual patient's length of stay to obtain the cost of care per patient per discharge.

111 All these steps were simplified by using the Clinical Cost Modelling Software Version 2.1 (CCM Ver. 2.1).

$112 \mathrm{CCM}$ is the step-down costing tool that is being used by the casemix system in UKMMC.

114 The cost is triangulated by developing clinical pathways for a hypoglycaemic episode. An expert group

115 meeting was held to develop the common clinical pathways of managing hypoglycaemia. These experts

116 include physicians, endocrinologists, and pharmacists who are working in the A\&E department and also in

117 the clinical departments of the hospital.

119 In imputing the national burden, estimates for incidence and prevalence of diabetes were obtained from

120 NMHS 2015 [8] and the International Diabetes Federation.[9] The possible episode of hypoglycaemia

121 incidence was estimated from the Malaysian Hypoglycaemia Assessment Tool Study (HAT study) [16].

122 The unit cost for management of hypoglycaemia calculated in the step-down approach was used to estimate

123 the burden. Sensitivity analysis was carried out by varying the incidence of hypoglycaemia to obtain the

124 worst-case scenario and best-case scenario. 


\section{Results}

131903 cases $(0.54 \%$ of the total cases) discharged from UKMMC between January 2010 and September 2015

132 had a diagnosis of hypoglycaemia (Table 1).

133 Table 1. Number of Hypoglycaemia Patients By Year in UKMMC Casemix Database

\begin{tabular}{cccc}
\hline Year & Nos. & Total Discharges & \% Hypoglycaemia of Total Discharges \\
\hline 2010 & 199 & 32,144 & $0.62 \%$ \\
2011 & 157 & 26,262 & $0.60 \%$ \\
2012 & 180 & 28,728 & $0.63 \%$ \\
2013 & 177 & 33,473 & $0.53 \%$ \\
2014 & 119 & 27,303 & $0.44 \%$ \\
2015 & 71 & 18,623 & $0.38 \%$ \\
\hline Total & 903 & 166,533 & $0.54 \%$
\end{tabular}

136 Among these cases, 33.4\% were classified into MY-DRG ${ }^{\circledR}$ casemix groups of Endocrine, Nutrition and

137 Metabolism Group followed by Respiratory System Group (12.6\%) and Cardiovascular System Group

138 (9.7\%).Central Nervous System and Nephro-urinary System contributed 6.4\% of the hypoglycaemia

139 individually (Table 2).

141 Table 2. Hypoglycemia by Casemix Main Group (CMG)

\begin{tabular}{clcc}
\hline No & Casemix Main Groups (CMG) & Frequency & Percent \\
\hline 1 & Endocrine system, nutrition \& metabolism Groups & 302 & 33.4 \\
2 & Respiratory system Groups & 114 & 12.6 \\
3 & Cardiovascular system Groups & 88 & 9.7 \\
4 & Central nervous system Groups & 58 & 6.4 \\
5 & Nephro-urinary System Groups & 58 & 6.4 \\
6 & Infectious \& parasitic diseases Groups & 51 & 5.6 \\
7 & Musculoskeletal system \& connective tissue Groups & 47 & 5.2
\end{tabular}


9 Hepatobiliary \& pancreatic system Groups

10 Female reproductive system Groups

11 Skin, subcutaneous tissue \& breast Groups

12 Haemopoeitic\& immune system Groups

13 Ear, nose, mouth \& throat Groups

14 Myeloproliferative system \& neoplasms Groups

15 Mental Health and Behavioral Groups

16 Injuries, poisonings \& toxic effects of drugs Groups

17 Eye and Adnexa Groups

18 Male reproductive System Groups

19 Deliveries Groups

Factors influencing health status \& other contacts with health services Groups

21 Substance abuse \& dependence Groups

144 Most of the hypoglycaemic conditions were admitted to the medical ward (91.7\%), and 8.1\% were

145 admitted to the surgical unit.

147 Of the 302 hypoglycaemic cases with endocrine problems, diabetes in particular, 244 cases were admitted

148 primarily for hypoglycaemia and 58 were admitted for hypoglycaemia as a secondary reason (Table 3). 
Table 3. Type of Hypoglycemia among cases in CMG Endocrine System, Nutrition \& Metabolism

\begin{tabular}{lcc}
\hline Type of Hypoglycaemia & Frequency & Percent \\
\hline Hypoglicaemia as main diagnosis & 244 & $80.8 \%$ \\
Hypoglicaemia as secondary diagnosis & 58 & $19.2 \%$ \\
\hline Total & 302 & 100
\end{tabular}

Only 244 cases with hypoglycaemia as primary reason were included in the study for further analysis.

158 Females were more likely to suffer from hypoglycaemia, as almost 52\% of the cases were female. $99.2 \%$ of

159 all cases were discharged home well. In the MY-DRG ${ }^{\circledR}$ system, patients are classified into one of the three

160 levels of severity. Cases in severity level 1 do not have complications or co-morbidities where as those in

161 severity level 2 have minor complications and co-morbidities. Patients in severity level 3 have major

162 complications and co-morbidities. About $57.4 \%$ of the primary hypoglycaemia cases were in severity level

163 3. Most (88.1\%) of the patients suffering from hypoglycaemic attacks were in the age group of 50-79 years

164 old, with $55.3 \%$ of the cases required not more than 5 days of hospital admission (Table 4). 
177 Table 4: Characteristics of hypoglycemic cases among CMG Endocrine System, Nutrition and Metabolism

\begin{tabular}{|c|c|c|}
\hline \multicolumn{2}{|l|}{ Characteristics } & \multirow{2}{*}{$\begin{array}{l}\text { Hypoglycemia as primary diagnosis } \\
(\mathrm{n}=244) \\
127(52 \%)\end{array}$} \\
\hline Gender (Female, $\%$ & & \\
\hline \multirow[t]{2}{*}{ Discharged status } & - Discharged to home & $242(99.2 \%)$ \\
\hline & - Others & $2(0.8 \%)$ \\
\hline \multirow[t]{3}{*}{ Severity Level } & - Severity Level 1 & $23(9.4 \%)$ \\
\hline & - Severity Level 2 & $81(33.2 \%)$ \\
\hline & - Severity Level 3 & $140(57.4 \%)$ \\
\hline \multirow[t]{6}{*}{ Age group } & - $20-29$ years & $3(1.2 \%)$ \\
\hline & - 30 - 39 years & $8(3.3 \%)$ \\
\hline & - 40 - 49 years & $18(7.4 \%)$ \\
\hline & - 50 - 59 years & $34(13.9 \%)$ \\
\hline & - 60 - 69 years & $84(34.4 \%)$ \\
\hline & - 70 - 79 years & $97(39.8 \%)$ \\
\hline \multirow[t]{6}{*}{ Length of stay } & $-<=5$ days & $135(55.3 \%)$ \\
\hline & - $6-10$ days & $77(31.6 \%)$ \\
\hline & - $11-15$ days & $18(7.4 \%)$ \\
\hline & $-16-20$ days & $10(4.1 \%)$ \\
\hline & - $21-25$ days & $3(1.2 \%)$ \\
\hline & - $26-30$ days & $1(0.4 \%)$ \\
\hline
\end{tabular}

179 The cost for a patient to be treated at UKMMC A\&E department for hypoglycaemia is RM 741 (USD 190).

180 Generally, the cost of hypoglycaemic cases admitted to the medical unit is less costly than other units: RM

181 1,375 (USD 352) at medical unit compared toRM 1,679 (USD 430) for obstetrics and gynaecology unit,

182 and (RM 2,611 (USD 668) atsurgical unit. Out of 903 cases with hypoglycaemia as diagnosis, 828 cases

183 are from medical ward, 73 are from surgical ward and only 2 are from O\&G unit. 
185 As an average, the cost of care for hypoglycaemia varies with severity level. Although the mean cost of

186 care for hypoglycaemia at severity 1 and 2 is RM7,054 (USD 1,804) and RM7,333 (USD 1,875)

187 respectively, the mean cost reaches RM10,401 (USD 2,660) when the condition becomes severity level 3.

188 The median cost for cases diagnosing hypoglycaemia as the primary diagnosis was RM 6,875) (USD

1891,758 ) and cases of hypoglycaemia as secondary diagnosis was found to be RM 11,000 (USD 2,813) The

190 median length of stay for hypoglycaemic cases was 5 days for primary diagnosis and 8 days for secondary

191 diagnosis. In this study, the focus is on the diabetic cases with hypoglycaemia as primary reason for

192 admission and the median cost found was RM 6,875) (USD 1,758) with a median length of hospital stay of

1935 days (Table 5).

Table 5. The cost (RM) of Hypoglycemia by diagnosis type and severity level

\begin{tabular}{|c|c|c|c|c|c|c|c|c|}
\hline $\begin{array}{l}\text { Type of } \\
\text { Hypoglycaemia }\end{array}$ & Severity & $\mathrm{N}$ & $\begin{array}{l}\text { Mean } \\
(\mathrm{RM})\end{array}$ & $\begin{array}{l}\mathrm{SD} \\
(\mathrm{RM})\end{array}$ & $\begin{array}{l}\text { Med } \\
(\mathrm{RM})\end{array}$ & $\begin{array}{l}\text { Min } \\
(\mathrm{RM})\end{array}$ & $\begin{array}{l}\text { Max } \\
(\mathrm{RM})\end{array}$ & $\%$ \\
\hline \multirow{4}{*}{$\begin{array}{l}\text { Hypoglycaemia a } \\
\text { main diagnosis }\end{array}$} & Severity Level-1 & 23 & 7,054 & 3,807 & 5,500 & 2,750 & 15,125 & $9.3 \%$ \\
\hline & Severity Level-2 & 81 & 7,333 & 4,165 & 6,875 & 2,750 & 26,125 & $32.8 \%$ \\
\hline & Severity Level-3 & 140 & 10,401 & 7,346 & 8,250 & 2,750 & 36,554 & $57.9 \%$ \\
\hline & $\overline{\text { Total }}$ & 244 & 9,083 & 6,363 & 6,875 & 2,750 & 36,554 & $100.0 \%$ \\
\hline
\end{tabular}

200 The cost by different cost centres was explored. $30 \%$ of the cost came from the final cost centre (the ward

201 services cost), $16 \%$ of the cost came from ICU services and $15 \%$ from pharmacy and drug services.

203 Using the information from International Diabetes Federation [8], the HAT study [16] and the NMHS 2015

204 [9], the assumption on incidence and prevalence were made. From the NMHS 2015, 17.5\% of adult

205 Malaysians are estimated to have diabetes mellitus, with $8.3 \%$ of them with known diabetes. $25.1 \%$ of the

206 known diabetics are on insulin therapy, which is equivalent to 404,619 people. Using a conservative 
assumption, the HAT study estimated that among type 2 DM patients, the annual incidence of any type of hypoglycaemic episode is $47.1 \%$ and severe hypoglycaemia is $16.8 \%$. [16] Based on the assumptions on

209 the number of cases requiring hospital admission and the mean cost for hypoglycaemia as main diagnosis

210 in UKMMC for severity level 1 to 3, the calculation was developed as base case scenario, best case

211 scenario and worst case-scenario.We estimated the worst case scenario based on the retrospective arm

212 findings of the HAT Studywhich is $5.9 \%$ of severe hypoglycaemia cases (23,872 patient) assumed to

213 require hospital admission. The base case scenario is calculated based on an expert group discussion with

214 local clinicians, which reached a consensus of 3.2\% prevalence (12,948 patients) for hypoglycaemia-related

215 hospital admissions. In inthe best case-scenario, $2.5 \%$ prevalence (10,115 patients) was used, from the

216 prospective arm findings of the HAT Study on hypoglycaemia hospital admissions. We assume that all

217 these cases require hospital admission for at least once.

218 The total cost of care for hypoglycaemia among adult diabetes in Malaysia was estimated to be RM 117.4

219 (USD 30.0) million, approximately $0.5 \%$ of Ministry of Health annual budget allocation of RM 22.16

220 (USD 5.67) billion in 2014. [17] The national economic burden estimates based on the bestcase-scenario

221 and the worstcase-scenario range from RM 91.7 (USD 23.5) million to RM 216.5 (USD 55.4) million.

222 (Table 6)

\section{Table 6. The Estimated Cost and Economic Burden of Hypoglycemia}

\begin{tabular}{llllll}
\hline $\begin{array}{l}\text { Hypoglycemia as primary } \\
\text { diagnosis }\end{array}$ & $\begin{array}{l}\text { \% of } \\
\text { cases }\end{array}$ & $\begin{array}{l}\text { Mean cost } \\
\text { per } \\
\text { admission } \\
(\mathrm{RM})\end{array}$ & $\begin{array}{l}\text { Worst case scenario } \\
(5.9 \% \text { admission })\end{array}$ & $\begin{array}{l}\text { Base scenario; } \\
(3.2 \% \text { Admission })\end{array}$ & $\begin{array}{l}\text { Best case scenario } \\
(2.5 \% \text { Admission })\end{array}$ \\
\hline Severity Level 1 & 9.4 & 7,054 & $15,829,227$ & $8,585,371$ & $6,707,321$ \\
Severity Level 2 & 33.2 & 7,333 & $58,118,921$ & $31,522,127$ & $24,626,662$ \\
Severity Level 3 & 57.4 & 10,401 & $142,522,937$ & $77,300,576$ & $60,391,075$ \\
\hline Total Cost & & 9,083 & $216,471,136$ & $\mathbf{1 1 7 , 4 0 8 , 0 7 4}$ & $91,725,057$ \\
\hline $\begin{array}{l}\text { \% MOH Budget } \\
\text { RM 22,160,380,300) }\end{array}$ & & & & $\mathbf{0 . 5}$ & 0.4 \\
\hline
\end{tabular}




\section{Discussion}

232 Proper glycaemic control is required to minimize the risk of microvascular complication of diabetes among

233 diabetics. At the same time, diabetes management commonly result in hypoglycaemic episodes. The

234 occurrence of hypoglycaemia is widely variable. The frequency of severe hypoglycaemia requiring

235 emergency services in patients with type 2 DM receiving insulin therapy is comparable to type $1 \mathrm{DM}$

236 depending on how diabetes is being managed. Incidence rates were 11.5 and 11.8 events per 100 patient-

237 years for type 1 and type 2 patients treated with insulin, respectively [17]. A study in Canada stated that

$2381.9 \%$ of individuals had at least one hypoglycaemia related A\&E visits, and $0.1 \%$ was admitted to hospital.

239 In terms of incidence rate, 5.2 cases and 0.3 cases per 1000 patient-year required A\&E visits and hospital

240 admission respectively [18]. In the HAT study, hypoglycaemic episodes of hospital admissions among DM

241 cases in Malaysia were measured both prospectively and retrospectively. Findings from the prospective and

242 retrospective component of the HAT study were reviewed and the most conservative estimates was selected

243 and subsequently validated by specialists from different hospitals in Malaysia. $47.1 \%$ of the type II DM

244 cases with insulin experienced any type of hypoglycaemia and16.8\% had severe hypoglycaemic episodes

245 within a 6-month period [16]. Based on their clinical experience, the expert group of specialists determined

246 a 3.2\% prevalence for hypoglycaemia requiring at least one hospital admission. The incidence of hospital

247 admission was conservatively estimated as 3.2 episodes per person year and also estimated with the worst

248 case-scenario with 10 episodes per person per year. Even with the best-case scenario, the incidence of

249 admission due to hypoglycaemic episodes is significantly higher than elsewhere [18, 19].

251 Several investigations are required to confirm and manage hypoglycaemia cases appropriately. Based on

252 severity and management protocol, the duration of hospital stay may vary from place to place. The cost of

253 hospital care is also dependent on how long the patient was admitted to the hospital. The mean length of

254 hospital stay ranged from 5.5 to 9.8 days in most studies (20-22). The median length of stay is 4 days in the

255 Canada [19]. The findings from our study also provide similar estimates with the mean length of hospital

256 stay was 6.5 days and the median length of stay was 5 days. Due to the wide variation and huge standard

257 deviation, the median length of stay was selected as the common length of hospital stay in this study. 
259 Depending on the level of severity, a hypoglycaemic condition may be treated at home, at A\&E department

260 or at hospital wards. The cost of care also varies with the level of severity. Our study showed that the cost

261 of care at A\&E department was RM 741 (USD 190) per case. On the other hand, the mean cost of care for a

262 patient admitted for hypoglycaemia is RM 9,083 (USD 2,323).

264 The HAT study presented the proportion of patients admitted to hospital, but not the patients requiring

265 A\&E visit prior to hospital admission. Other country studies stated that the proportion of hypoglycaemia-

266 related hospital admissions after the treatment in Emergency Department (ED) varies between 11-28\% [18,

267 23]. We do not have local estimates for this information but based on our expert group review, it could be

268 as high as $50 \%$. However, we used $5.9 \%$ of the cases require hospital admission as the worst case-scenario

269 and $2.5 \%$ as the best case-scenario from the findings in the Malaysian HAT Study [16].

271 Regardless of hypoglycaemia-related hospitalization is seemingly low, the actual number of hypoglycaemic

272 episodes requiring hospital care can impose both clinical and economic burden. In this study we have

273 focused more on the cost of care for severe episodes of hypoglycaemia at tertiary academic institutions and

274 the total economic burden introduced by hypoglycaemia. Comparing the economic cost of hypoglycaemia

275 is difficult due to the differences in health care systems and also in defining hypoglycaemia itself. Costing

276 studies from the literature showed that hypoglycaemia admissions in Scotland costs USD 303 (£218) per

277 person per day [22] whereas in Canada, where an average cost of USD 7,000 is required for an average

278 hospitalization due to hypoglycaemia for 7 days [19]. nIn case of Thailand, a patient with hypoglycaemia

279 requires 6 days of hospital stay as an average requiring nearly USD 700 (THB 22,000) per episode. [24] A

280 recent study in Korea estimated the medical costs for a hypoglycaemic event ranged from USD 17.28 to

281 USD 1,857 at secondary and tertiary hospitals [25].

283 Although the individual cost of care in Malaysia is not significantly high compared to other countries, the

284 number of episodes requiring health care services at the hospital is considerably higher, making the total

285 cost of care higher. This brings to an estimated cost of care for hypoglycaemia among type II DM patients 
287 Germany for example, the estimated annual direct cost of severe hypoglycaemia by Type II DM during

288 1997-2000 was USD 54,980 (€ 44,338) per 100,000 inhabitants (26,27), and in comparison, this study

289 found the cost estimate to be significantly higher in Malaysia, amounting to at least USD 526,585 (RM

$2902,058,963)$ per 100,000 inhabitants. Although the length of stay and the unit cost of care are not necessarily

291 higher, the number of admission required determined the possible burden for hypoglycaemic care at

292 hospitals.

294 A significant portion of hypoglycaemic episodes are treated at home without the assistance of medical

295 services either at the A\&E or hospital admission [28], indicating that this study measures only the burden

296 visible at the tip of the iceberg. Higher frequency of hypoglycaemic events could also have significant

297 impact on quality of life as well as imposing indirect cost by limiting work capacity and work productivity.

\section{Conclusion}

300 The findings of this study showed that severe hypoglycaemia in patients with diabetes impose significant

301 impact on resource utilization. Regardless of seemingly a simple condition, hypoglycaemia can result in

302 substantial economic burden for national health care system. Preventing hypoglycaemic episode should be

303 included in diabetic management programs that emphasize on proper diabetic management and health

304 education. This could minimize hypoglycaemicrisk, which may lead to reducing overall health spending,

305 minimizing the fear of hypoglycaemia episodes and improving the compliance of diabetes management.

306 The high cost of hypoglycaemia management calls for a personalized approach to glycaemic control and

307 development of better guidelines for clinical decision making in diabetes control strategies. 


\section{Acknowledgement}

318 We would like to acknowledge the financial support we received from Novo Nordisk Pharma (Malaysia)

319 Sdn Bhd to undertake this study. We would also like to extend our gratitude to the Research and Ethics

320 Committee of National University of Malaysia for providing the ethical approval to conduct this research.

321 We would also like to acknowledge Dr LetchumanRamanathan (Hospital Raja PermaisuriBainun), Prof Dr

322 Nor AzmiKamaruddin (UniversitiKebangsaan Malaysia), Dr Zanariah Hussein (Hospital Putrajaya) for

323 their valuable inputs in the estimated prevalence of hospital-admitted hypoglycaemia in Malaysia.

\section{Contributions}

326 SM Aljunid Aniza Ismail and Yin New Aung designed the study and prepare the first draft of the

327 manuscript. Siti Zafirah Abdul Rashid and Amrizal N Nur conducted the data analysis and prepare the

328 tables. SM Aljunid, Julius Cheah and Priya Matzen reviewed that first draft and finalised the manuscript for

329 publication 


\section{$331 \quad$ References}

332 1. Cryer PE. Glucose homeostatis and hypoglycemia. 10th ed. USA: Saunders/Elsevier, 2003.

333 2. Shafiee G, Mohajeri-Tehrani M, Pajouhi M, et al. The importance of hypoglycemia in diabetic 334 patients. Journal of Diabetes and Metabolic Disorders. 2012; 11: 17-17.

335 3. Sircar M, Bhatia A, Munshi M. Review of Hypoglycemia in the Older Adult: Clinical Implications 336 and Management. Can J Diabetes. 2016; 40: 66-72.

337 4. McCrimmon RJ, Sherwin RS. Hypoglycemia in Type 1 Diabetes. Diabetes. 2010; 59: 2333-39.

338 5. Cryer PE. Diverse Causes of Hypoglycemia-Associated Autonomic Failure in Diabetes. New 339 England Journal of Medicine. 2004; 350: 2272-79.

340 6. Miller CD, Phillips LS, Ziemer DC, et al. Hypoglycemia in patients with type 2 diabetes mellitus. 341 Archives of internal medicine. 2001; 161: 1653-9.

342 7. Jonsson L, Bolinder B, Lundkvist J. Cost of hypoglycemia in patients with Type 2 diabetes in 343 Sweden. Value in health : the journal of the International Society for Pharmacoeconomics and Outcomes 344 Research. 2006; 9: 193-8.

345 8. Pramming S, Thorsteinsson B, Bendtson I, et al. Symptomatic hypoglycaemia in 411 type 1 346 diabetic patients. Diabetic medicine: a journal of the British Diabetic Association. 1991; 8: 217-22.

347 9. Jorgensen HV, Pedersen-Bjergaard U, Rasmussen AK, et al. The impact of severe hypoglycemia 348 and impaired awareness of hypoglycemia on relatives of patients with type 1 diabetes. Diabetes care. 2003; 349 26: 1106-9.

350 10. International Diabetic Federation I. IDF Diabetes Atlas. 7th ed., 2015.

351 11. Ministry of Health M. National Health Morbidity Survey. 2015.

352 12. OECD, WHO. Health at a Glance: Asia/Pacific 2014. OECD Publishing, 2014.

353 13. Institue for Public Health Minitry of Health Malaysia. National health and morbidity Survey 354 (NHMS) 2015. 2015.

355 14. Mafauzy BM. Diabetes Mellitus in Malaysia. Journal of Medicine Malaysia. 2006; 61: 2.

356 15. American Diabetes Association A. Standards of Medical Care in Diabetes-2011. Diabetes care. 2011; 357 34: S11-S61.

358 16. Hussein et al. Hypoglycemia awareness among insulin-treated patients with diabetes in Malaysia: 359 A cohort subanalysis of the HAT study. Diabetes Research and Clinical Practice. 2017; 133: 40-49

$360 \quad$ 17. Ministry of Health. Annual Report. 2014. 
361 18. Leese GP, Wang J, Broomhall J, et al. Frequency of severe hypoglycemia requiring emergency treatment in type 1 and type 2 diabetes: a population-based study of health service resource use. Diabetes care. $2003 ; 26: 1176-80$.

364 19. Mosian J, Breton M-C, Villeneuve J, et al. Hypoglycemia-Related Emergency Department Visits 365 and Hypoglycemia-Related Hospitalizations among New Users of Antidiabetes Treatments. Canadian 366 Journal of Diabetes. 2013; 37: 143-49.

367 20. Bloomfield HE, Greer N, Newman D, et al. Predictors and Consequences of Severe Hypoglycemia

368 in Adults with Diabetes - A Systematic Review of the Evidence. Department of Veterans Affairs, 2012.

369 21. McEwan P, Larsen Thorsted B, Wolden M, et al. Healthcare resource implications of

370 hypoglycemia-related hospital admissions and inpatient hypoglycemia: retrospective record-linked cohort 371 studies in England. BMJ Open Diabetes Research \& Care. 2015; 3.

372 22. Amiel SA, Dixon T, Mann R, et al. Hypoglycaemia in Type 2 diabetes. Diabetic Medicine. 2008; 373 25: 245-54.

374 23. Brackenridge A, Wallbank H, Lawrenson RA, et al. Emergency management of diabetes and 375 hypoglycaemia. Emergency medicine journal : EMJ. 2006; 23: 183-5.

376 24. Riewpaiboon A, Chatterjee S, Piyauthakit P. Cost analysis for efficient management: diabetes 377 treatment at a public district hospital in Thailand. The International journal of pharmacy practice. 2011; 19: $378 \quad 342-9$.

379 25. Kim G, Lee YH, Han MH, et al. Economic Burden of Hypoglycemia in Patients with Type 2 380 Diabetes Mellitus from Korea. PloS one. 2016; 11: e0151282.

381 26. Holstein A, Patzer OM, Machalke K, et al. Substantial increase in incidence of severe 382 hypoglycemia between 1997-2000 and 2007-2010: a German longitudinal population-based study. Diabetes 383 care. 2012; 35: 972-5.

384 27. Holstein A, Plaschke A, Egberts E-H. Incidence and Costs of Severe Hypoglycemia. Diabetes 385 care. 2002; 25: 2109-10.

386 28. Frier BM. Hypoglycaemia in the diabetic adult. Bailliere's clinical endocrinology and metabolism. 387 1993; 7: 757-77. 